\title{
Determination of positions and curved transition pathways of screw dislocations in BCC crystals from atomic displacements
}

\author{
R. Gröger \\ Central European Institute of Technology - Institute of Physics of Materials (CEITEC IPM), \\ Academy of Sciences of the Czech Republic, Žižkova 22, 61662 Brno, Czech Republic \\ V. Vitek \\ Department of Materials Science and Engineeing, University of Pennsylvania, \\ 3231 Walnut Street, Philadelphia, PA 19104, USA
}

\begin{abstract}
The theoretical description of the thermally activated dislocation glide in pure crystals depends crucially on the shape of the Peierls barrier that the dislocation has to overcome when moving through the lattice. While the height of this barrier can be obtained using saddle-point search algorithms such as the Nudged Elastic Band (NEB) method, its exact shape depends on the details of the approximation of the transition pathway of the system. The purpose of this paper is to formulate a procedure that allows to identify the dislocation positions along the path directly from the displacements of atoms in its core. We investigate the performance of this model by calculating curved paths of the $1 / 2[111]$ screw dislocation in tungsten modeled by a Bond Order Potential using a series of images obtained by employing a modified NEB method at zero applied stress and for positive/negative shear stresses perpendicular to the slip direction. The Peierls barriers resulting from the curved paths are shown to be substantially different from those obtained when assuming straight dislocation path. For both straight and curved dislocation pathways we calculate the temperature dependencies of the flow stress and compare these predictions with direct experimental measurements. We show that a significantly better agreement with experiments is obtained if the curved dislocation pathway is taken into account.
\end{abstract}

Keywords: screw dislocation, BCC metal, dislocation pathway, Peierls barrier, Nudged Elastic Band. PACS: 61.72.Hh, 02.60.-x, 45.10.Db

\section{Introduction}

$1 / 2\langle 111\rangle$ screw dislocations control the plastic deformation of pure body-centered cubic (BCC) metals. Atomistic studies of the structure and glide of these dislocations have been made using a wide variety of interatomic potentials and ab initio methods [1-11]. In calculations of the glide of dislocations under the effect of applied stresses, the evolution of the system is viewed as a coordinated motion of $N$ atoms along an a priori unknown path in the configurational space spanned by the $3 N$ degrees of freedom (DOF). Defining the state of the system in the full $3 N$ dimensional space presents severe difficulties when developing theoretical models of thermally activated glide of dislocations [12-14], which serve to coarse-grain the atomistic results to the standard continuum theory of dislocations [15] in which the position of the dislocation is well-defined. Not surprisingly, there has been a long-standing interest in developing approximate schemes to extract the effective position of the dislocation from the coordinates of atoms obtained by molecular statics simulations

Email address: groger@ipm.cz (R. Gröger)

Preprint submitted to Elsevier

July 8, 2015

(C) 2015. This manuscript version is made available under the Elsevier user license

http://www.elsevier.com/open-access/userlicense/1.0/ 
of single dislocations. This approach cannot be used without developing a systematic procedure that maps the $3 N$ atomic DOF to the position of the dislocation.

The most obvious choice that leads to the reduction of complexity of the system is to suppose that the dislocation moves between two neighboring sites along the straight line connecting these sites. This has been implicitly assumed in most papers employing the Nudged Elastic Band (NEB) method [16, 17], where the position of the dislocation along the minimum energy path scales linearly with the image number $[7,11]$. Whereas the obtained barrier can be used to assess the stability of each intermediate state along the path, it does not constitute the Peierls barrier that could be used to develop models of thermally activated dislocation glide such as those due to Celli et al. [12] and Dorn and Rajnak [13]. The reason is that a straightforward application of the NEB method to all DOF in the system leads to nonuniform distributions of dislocation positions among the images, which affects the shape of the obtained Peierls barrier if plotted along a straight line between two neighboring minimum-energy positions in the lattice [18]. It should be emphasized that not only the height of the Peierls barrier but its entire shape enters the models of thermally activated dislocation glide. A significant improvement of the calculation of the Peierls barrier is obtained using our modification of the NEB method [18], where relaxations of atoms in the block are taken into account at every step of the method. This method called NEB $+\mathrm{r}$ (a shorthand for the NEB method with relaxations) guarantees that the positions of the dislocation when following the minimum energy path are distributed uniformly among the images and thus the assumed proportionality between the dislocation position and the image number is justified. These developments have led to an accurate estimate of the Peierls barrier of $1 / 2\langle 111\rangle$ screw dislocations in BCC W and its changes under the applied stress [18, 19]. However, these calculations are still based on the assumption that the path of the dislocation between two neighboring images is a straight line, which is not true in general.

In principle, it should be possible to deduce the dislocation position (and thus also its path between two positions in the lattice) directly from the displacements of atoms as obtained from molecular statics calculations or from the NEB (NEB+r) methods. This idea dates back to Peierls and Nabarro [20, 21], who associated the dislocation position with the point in the slip plane at which the displacement parallel to the slip direction, interpolated from the displacements of atoms, is equal to $b / 2$, where $b$ is the magnitude of the Burgers vector of the dislocation. Since then, this argument was used many times. In particular, it was adopted by Pizzagalli et al. [8], Rodney and Proville [10] and Proville et al. [22], where the position of the dislocation is defined by a single coordinate corresponding to the distance that the dislocation makes in a well-defined slip plane. The same level of approximation was also used by Ventelon et al. [23] in one of their methods (disregistry function method) that uses a combination of isotropic elasticity and geometry of the BCC lattice to define the position of the dislocation.

However, the movement of the dislocation should be viewed in general as a two-dimensional event during which the center of the dislocation translates along a curved path. In attempt to resolve this path, Ventelon et al. [23] also proposed another model, whereby the dislocation position is identified using a cost function that is based on both the actual positions of atoms (as obtained from atomistic simulations) and the positions of atoms determined by anisotropic elasticity for some trial dislocation position in the $\{111\}$ plane. The actual position of the dislocation is then obtained by minimizing this cost function that is defined as the distance between the two sets of coordinates in the five-dimensional subspace spanned by the coordinates of the five most displaced atomic columns around the dislocation. Since the position of the dislocation is determined by the relative displacements of the three atomic columns closest to the dislocation in the direction parallel to the Burgers vector, a similar approach can be devised that is based on inversion of the Eshelby-Stroh sextic formalism (see, for example Hirth and Lothe [24]), which provides elastic displacements of atoms corresponding to a given position of the dislocation. We have investigated this possibility earlier (unpublished work). This approximation can be used when the dislocation is positioned centrally in between the nearest three $\langle 111\rangle$ atomic columns, but it quickly worsens as the dislocation gets closer to any of these columns or the boundary between the neighboring dislocation sites.

A different scheme whereby the position of the 1/2[111] dislocation is determined by extrapolating differential displacements between the three atoms surrounding the dislocation when projected onto the (111) plane into the interior of this triangle was developed by Itakura et al. [25]. This approach is closely related to a purely geometrical concept of barycentric (or trilinear) coordinates known from ternary diagrams 
that was originally applied to estimate the position of the dislocation by Heinrich and Schellenberger [26]. This method makes use of actual positions of atoms, but expressing the dislocation position, projected onto the (111) plane, as a linear combination of the displacements of the three nearest atoms in the direction parallel to the Burgers vector is merely a convenient choice that is not fully justified physically. This approximation was avoided in the recent work of Dezerald et al. [27] who aimed to reconstruct the twodimensional Peierls barrier by interpolating the line energy of the dislocation, calculated by first principles, from two straight dislocation paths. One connects the neighboring potential minima in the $\{110\}$ plane and the other passes from an atom ("split-core" configuration) to the so-called "hard-core" position in the $\langle 110\rangle$ direction perpendicular to the first path. The Peierls potential is then expressed in the form of a Fourier series with the Fourier coefficients adjusted so as to minimize the least squares error between the calculated data and the Fourier series. The path of the dislocation between two neighboring minimumenergy dislocation sites in the $\{110\}$ plane is obtained using the disregistry and cost function methods of Ventelon et al. [23]. Although these paths are smooth, the corresponding Peierls barriers display sharp maxima for BCC Mo, $\mathrm{W}$ and $\mathrm{Nb}$, which are somewhat less pronounced for $\mathrm{BCC}$ Ta and $\mathrm{V}$ as well as for ferromagnetic BCC Fe. This does not agree with the analysis of Suzuki et al. [28] and our more recent studies (Refs. [14, 29]), which show that in order to reproduce the experimentally measured temperature dependence of the flow stress, the Peierls potential has to possess a flat maximum.

In this paper, we develop a procedure that specifies the position of the 1/2[111] screw dislocation in BCC crystals (and thus the curved dislocation pathway) using solely the actual displacements of atoms in the dislocation core, without invoking isotropic or anisotropic elasticity. It generalizes the concept established by Peierls and Nabarro $[20,21]$ in that it considers all three $\{110\}$ planes on which the dislocation can move. These calculations provide three lines whose intersection defines the position where the dislocation intersects the (111) plane. We demonstrate the performance of this method by calculating the paths of the straight $1 / 2[111]$ screw dislocation in BCC W in the three possible $\{110\}$ planes from the discrete snapshots (images) of the system obtained using NEB $+\mathrm{r}$ calculations [19]. These calculations are made under zero applied stress and for positive and negative shear stresses perpendicular to the slip direction. We demonstrate that the shape of the Peierls barrier changes significantly when the transition pathway of the dislocation is curved as compared to that obtained by assuming the straight path between two adjacent minimum-energy configurations in the lattice [19]. To demonstrate the accuracy of this model, we predict the temperature dependence of the flow stress for loading in tension along [149] that was measured experimentally by Brunner [30]. The theoretical predictions agree very well with the experimental data once the curved pathway of the dislocation is taken into account, whereas the agreement for the straight dislocation path is tentative at best.

\section{Complexity reduction}

Let us consider that $\mathbf{X}_{i}$ is the position of the atom $i$ in the perfect lattice. Upon inserting the 1/2[111] screw dislocation, imposing additional displacements due to the externally applied stress tensor $\boldsymbol{\Sigma}$ and relaxing the atoms, every atom $i$ moves into its new position, $\mathbf{x}_{i}^{0}$, where $i=1,2, \ldots, N$ are numbers of atoms. This configuration corresponds to the stable equilibrium with the energy $E\left(\mathbf{x}_{1}^{0}, \ldots, \mathbf{x}_{N}^{0} ; \boldsymbol{\Sigma}\right)$. In this configuration the dislocation is at the bottom of the Peierls valley for the applied stress $\boldsymbol{\Sigma}$. We will now consider that the dislocation has moved away from this minimum as a straight line at a constant applied stress $\boldsymbol{\Sigma}$, which was accomplished by displacing the atoms from $\mathbf{x}_{i}^{0}$ to $\mathbf{x}_{i}$. The energy of this new (generally nonequilibrium) configuration will be denoted $E\left(\mathbf{x}_{1}, \ldots, \mathbf{x}_{N} ; \boldsymbol{\Sigma}\right)$. The enthalpy of the final state of the system relative to its initial state per unit length of the dislocation (or, simply, the line enthalpy of the dislocation) is then

$$
H\left(\mathbf{x}_{1}, \ldots, \mathbf{x}_{N} ; \boldsymbol{\Sigma}\right)=\frac{E\left(\mathbf{x}_{1}, \ldots, \mathbf{x}_{N} ; \boldsymbol{\Sigma}\right)-E\left(\mathbf{x}_{1}^{0}, \ldots, \mathbf{x}_{N}^{0} ; \boldsymbol{\Sigma}\right)-W\left(\mathbf{x}_{1}-\mathbf{x}_{1}^{0}, \ldots, \mathbf{x}_{N}-\mathbf{x}_{N}^{0} ; \boldsymbol{\Sigma}\right)}{l_{\text {dislo }}}
$$

where $W$ is the work done by the applied stress $\boldsymbol{\Sigma}$ on shifting the atoms from $\mathbf{x}_{1}^{0}, \ldots, \mathbf{x}_{N}^{0}$ to $\mathbf{x}_{1}, \ldots, \mathbf{x}_{N}$, and $l_{\text {dislo }}$ the length of the dislocation segment contained in the simulated block. 
The left-hand side of (1) suggests that the state of the system is described by the $3 N$ DOF associated with the positions of all particles. However, this choice would not be practical from the computational point of view. As the dislocation moves through the lattice, the part of the energy that arises from the atoms close to the center of the dislocation varies much more than the long-range elastic energy contributed by the atoms far from the dislocation. It is thus more convenient to separate the atomic degrees of freedom into two classes: (i) the minimum number of DOF that determine the position of the dislocation, and (ii) all remaining DOF that do not affect the position of the dislocation and thus they represent merely the large-scale response of the system to the presence of the dislocation. For this purpose, we can use the concept of the Burgers circuit to identify the region of the atomic block that contains the dislocation in its interior. When viewing a BCC crystal along the [111] direction, the shortest of these circuits passes through three atoms that are closest to the center of the dislocation in the projection onto the (111) plane. This implies that only $9 \mathrm{DOF}$ are needed to describe the dislocation position, while the remaining $3 N-9$ DOF represent the displacements of other atoms in the block. However, the position of the dislocation is really determined by relative displacements of only three atoms in the direction of the Burgers vector. This reduces 9 DOF to three, which are not all independent because their differences must sum up to the magnitude of the Burgers vector. Therefore, the information about the position of the dislocation $\left(X_{D}, Y_{D}\right)$ is encoded in only two DOF.

These arguments suggest to find a procedure that maps the positions of atoms, $\mathbf{x}_{1}, \ldots, \mathbf{x}_{N}$, to the position of the intersection of the dislocation line with the $\{111\}$ plane perpendicular to the dislocation line in the perfect lattice (called hereafter the dislocation position), i.e.

$$
M:\left\{\mathbf{x}_{1}, \ldots, \mathbf{x}_{N}\right\} \mapsto\left(X_{D}, Y_{D}\right) .
$$

It is important to emphasize that $\left\{\mathbf{x}_{1}, \ldots, \mathbf{x}_{N}\right\}$ is defined in the deformed lattice with the dislocation, while $\left(X_{D}, Y_{D}\right)$ in the perfect lattice. If this mapping exists, the state of the system can be described using only two variables, the coordinates $\left(X_{D}, Y_{D}\right)$ of the intersection of the dislocation with the (111) plane of the perfect lattice. ${ }^{1}$ Hence, one could write the line enthalpy of the dislocation as

$$
H\left(X_{D}, Y_{D} ; \boldsymbol{\Sigma}\right)=H\left(\mathbf{x}_{1}, \ldots, \mathbf{x}_{N} ; \mathbf{\Sigma}\right),
$$

where the right-hand side is obtained from (1). Eq. (3) opens the possibility for developing a model of thermally activated dislocation glide, in which the transformation of the dislocation core is viewed as a motion of the center of the dislocation in the underlying Peierls potential.

For further developments, it will be convenient to introduce a transition pathway of the dislocation, $\xi$, that is defined by a series of discrete points $\left(X_{D}, Y_{D}\right)$ obtained from individual snapshots of the system as it moves along the minimum energy path. Hence, the left-hand side of $(3)$ is equivalent to $H(\xi ; \boldsymbol{\Sigma})$,

$$
H(\xi ; \boldsymbol{\Sigma}) \equiv H\left(X_{D}, Y_{D} ; \mathbf{\Sigma}\right)
$$

where $\xi$ represents a particular point $\left(X_{D}, Y_{D}\right)$ along a curved transition path of the dislocation. If the dislocation remains a straight line during this transition (which is the case at $0 \mathrm{~K}$ ), we may express the line enthalpy of the dislocation as

$$
H(\xi ; \boldsymbol{\Sigma})=V\left(\xi ; \boldsymbol{\Sigma}^{\text {nonglide }}\right)-\sigma^{\text {glide }} b \xi,
$$

where $V$ is the Peierls barrier ${ }^{2}$ and the second term is the work done by the applied stress on displacing the dislocation by the distance $\xi$ measured along the transition path. In equation (5), we have divided the applied stress as $\boldsymbol{\Sigma}=\boldsymbol{\Sigma}^{\text {glide }}+\boldsymbol{\Sigma}^{\text {nonglide }}$, where $\boldsymbol{\Sigma}^{\text {glide }}$ contains only the shear stress $\sigma^{\text {glide }}$ acting in the slip plane parallel to the slip direction (i.e. the Schmid stress), whereas $\boldsymbol{\Sigma}^{\text {nonglide }}$ contains all other stress components. The latter are all stress components that do not exert a Peach-Koehler force on the dislocation.

\footnotetext{
${ }^{1}$ We implicitly assume that all other DOF are relaxed upon specifying the dislocation position.

${ }^{2}$ The minimum of the Peierls barrier $V\left(\xi ; \boldsymbol{\Sigma}^{\text {nonglide }}\right)$ for a given applied stress tensor $\boldsymbol{\Sigma}^{\text {nonglide }}$ is shifted to zero.
} 
Clearly, the glide (Schmid) stress does work on displacing the dislocation, while non-glide stresses affect the shape of the Peierls barrier. The Peierls barrier can then be obtained from

$$
V\left(\xi ; \boldsymbol{\Sigma}^{\text {nonglide }}\right)=H(\xi ; \boldsymbol{\Sigma})+\sigma^{\text {glide }} b \xi .
$$

Here, the first term on the right-hand side is obtained directly from the NEB (NEB+r) calculations, as it is evident by combining (1), (3) and (4). The expression (6) is completely general and can be used to obtain the shape of the Peierls barrier for an arbitrary applied load. For the sake of simplicity, we will consider in the following that $\sigma^{\text {glide }}=0$. Hence, the NEB $(\mathrm{NEB}+\mathrm{r})$ calculations yield directly the line energies of the dislocation for different positions $\xi$ of the dislocation along the pathway, i.e. $V\left(\xi ; \boldsymbol{\Sigma}^{\text {nonglide }}\right)$, subject to a given non-glide stress tensor $\boldsymbol{\Sigma}^{\text {nonglide }}$.

\section{Position and curved path of the dislocation}

Upon inserting the dislocation parallel to the $z$ axis, applying external load and relaxing the atomic positions, the atoms move from their perfect lattice positions, $\mathbf{X}_{i}$, into their new positions, $\mathbf{x}_{i}$. These two sets of atomic coordinates can be used to obtain a differential displacement map that uniquely identifies the shortest Burgers circuit surrounding the dislocation. One such circuit is defined by the edges of the gray triangle in Fig. 1(a) with the dislocation at some unknown position in its interior. The three $\{110\}$ planes on which the dislocation can move are marked as $\alpha=1,2,3$ and distinguished by colors.

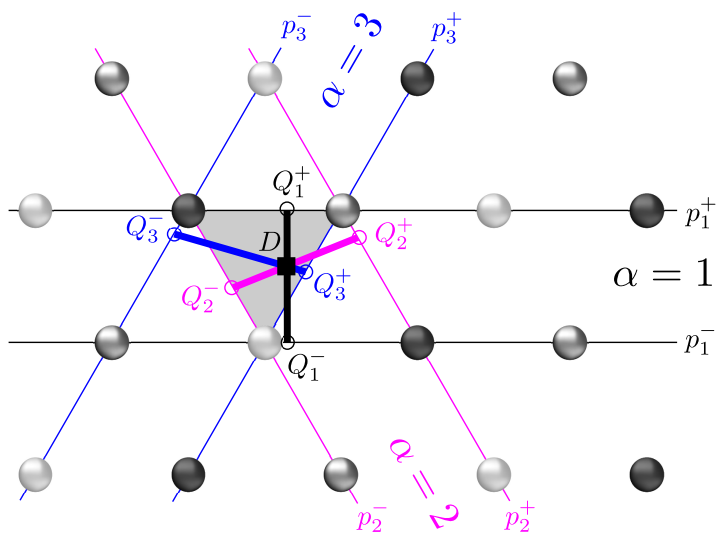

(a)

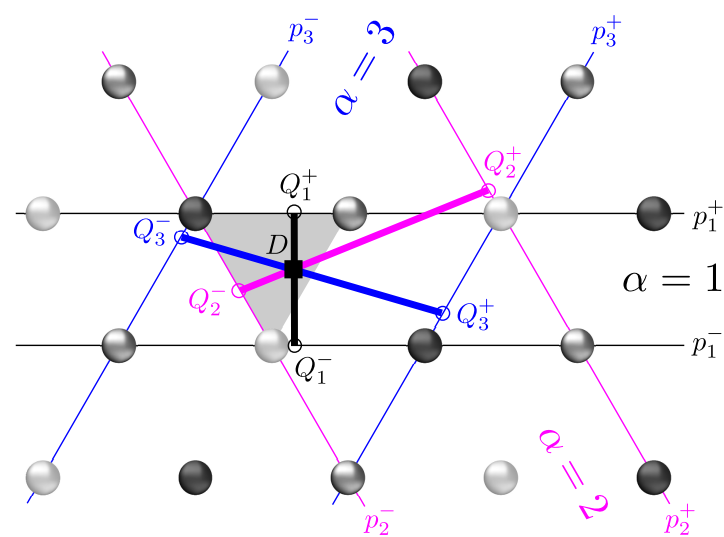

(b)

Figure 1: The three possible $\{110\}$ slip planes on which the dislocation can move in the lattice $(\alpha=1,2,3)$. In figure (a), the chains of atoms in the planes immediately above and below the triangle with the dislocation are marked $p_{\alpha}^{ \pm}$. The dislocation positions within these chains are marked $Q_{\alpha}^{ \pm}$. The sought position of the dislocation corresponds to the point $D$, which is defined as the intersection of the three line segments $Q_{\alpha}^{+} Q_{\alpha}^{-}$for $\alpha=1,2,3$. Figure (b) shows an alternative choice of the atomic chains from which the position of the dislocation can be calculated, which is used for all calculations in this paper made in Section 4 .

For each slip plane $\alpha$, we consider two planes $p_{\alpha}^{ \pm}$immediately above and below the dislocation site, which are drawn in Fig. 1 by thin lines. Each of these planes is represented by a finite number of atoms which are ordered so as to form a chain. The displacement of each atom from its position in the perfect BCC lattice, projected parallel to the Burgers vector of the dislocation, is then defined as

$$
u_{b}\left(p_{\alpha(i)}^{ \pm}\right)=\frac{\left[\mathbf{x}\left(p_{\alpha(i)}^{ \pm}\right)-\mathbf{X}\left(p_{\alpha(i)}^{ \pm}\right)\right] \cdot \mathbf{b}}{b},
$$

where $\mathbf{b}$ is the Burgers vector of the dislocation, and $b$ its magnitude. For the atoms far away from the dislocation core, the displacements $u_{b}$ approach constant values that represent the minimum and maximum of $u_{b}$ along the chain. Similarly as in Refs. [20,21], the position of the dislocation in each chain $p_{\alpha}^{ \pm}$, denoted 
hereafter $Q_{\alpha}^{ \pm}$, is assumed to coincide with the point along the chain, where the displacement in the direction parallel to the Burgers vector is half-way between its minimum and maximum,

$$
u_{b}\left(Q_{\alpha}^{ \pm}\right)=\frac{1}{2}\left[\min _{i} u_{b}\left(p_{\alpha(i)}^{ \pm}\right)+\max _{i} u_{b}\left(p_{\alpha(i)}^{ \pm}\right)\right] .
$$

For each possible slip plane $\alpha$, this equation provides two estimates of the dislocation position, one for the chain of atoms above $\left(Q_{\alpha}^{+}\right)$and the other below $\left(Q_{\alpha}^{-}\right)$the site with the dislocation. These points then define the line segments $Q_{\alpha}^{+} Q_{\alpha}^{-}$that are plotted in Fig. 1(a) by thick lines. The actual position of the dislocation is associated with the intersection of these line segments. This is marked in Fig. 1(a) as the point $D$. The implicit definition of $Q_{\alpha}^{ \pm}$using Eq. (8) is an approximation that, nevertheless, provides a good estimate of the position of the dislocation when it coincides with the centroid of the shaded triangle in Fig. 1(a), i.e. when the system is in equilibrium at zero applied shear stress parallel to the Burgers vector. This way of obtaining $Q_{\alpha}^{ \pm}$is adopted here for all states of the system along the minimum energy path irrespective of whether they represent equilibrium or nonequilibrium states.

It should be pointed out that there is no a priori guarantee that the three line segments $Q_{\alpha}^{+} Q_{\alpha}^{-}$for $\alpha=1,2,3$ will intersect at a single point. In general, one obtains three points that correspond to the intersections of three pairs of lines $Q_{\alpha}^{+} Q_{\alpha}^{-} \times Q_{\beta}^{+} Q_{\beta}^{-}$for the possible slip planes $\alpha \neq \beta$. This is illustrated for the dislocation moving in the (101) plane by blue in Fig. 2(a). Each small blue triangle was obtained by the procedure described above, while the solid curve is an approximation of the dislocation pathway obtained by connecting the centroids of the individual small triangles. For the image at the beginning and the end of the path, the three line segments in Fig. 1(a) intersect at a single point $D$ and thus the dislocation positions are determined uniquely. However, the size of the small triangles gets larger (and thus also the accuracy of this method quickly worsens) as the dislocation moves closer to the boundary between the neighboring dislocation sites.

The origin of the above-mentioned loss of accuracy can be unraveled by looking at the dependence of $u_{b}$ along the chain of atoms $p_{3}^{+}$, which is plotted in Fig. 2(b). For the equilibrium image at the beginning of the path $(I=0)$, i.e. when the dislocation is at the bottom of the Peierls valley, the displacement $u_{b}$ varies monotonously from its minimum at one end of the chain to the maximum at the other (black curve). In this case, the average displacement of atoms parallel to the Burgers vector is close to the inflection point of $u_{b}$. Provided the system is close to equilibrium, the position of the dislocation along the chain can thus be approximated using (8). This is, however, not the case for the image $I=5$ when 15 movable images are used. In this case, the bond between the two atoms in the core stretches by nearly $b / 2$, causing a sharp cusp along the chain, where the total displacement is larger than $b / 2$. This sudden increase of the relative displacements of atoms in the vicinity of the dislocation must be followed by a gradual decay of $u_{b}$ (negative relative displacements of atoms), eventually reaching a constant value dictated by the boundary conditions. This cusp does not appear instantaneously but, instead, develops gradually as the system moves out of equilibrium. This makes the estimates of the dislocation position using the procedure shown in Fig. 1(a) difficult even for the images relatively close to the equilibrium states. We have checked that the cusp appears in the images obtained from both NEB and NEB+r methods, using different BOPs as well as the AcklandThetford potentials [31]. It thus seems that the cusp is not specific to any description of bonding or the variant of the NEB method but, instead, it arises due to the crystallinity of the lattice.

To avoid the emergence of the cusp when the dislocation gets closer to the boundary between the neighboring dislocation sites, we have chosen different set of atomic chains along which the displacements $u_{b}$ are calculated. These are shown in Fig. 1(b), where the lines $p_{2}^{+}$and $p_{3}^{+}$are now moved one lattice step away from the shaded dislocation site. The same analysis as above was repeated using this choice of the three planes $p_{\alpha}^{ \pm}$and the obtained dislocation pathway is shown in Fig. 2(a) by red. One can see that the small triangles representing the nodes $Q_{\alpha}^{+} Q_{\alpha}^{-} \times Q_{\beta}^{+} Q_{\beta}^{-}(\alpha \neq \beta)$ are now very small and are nearly independent of the position of the dislocation. This increased accuracy follows directly from a monotonous character of the displacements $u_{b}$ along the chain $p_{3}^{+}$, shown in Fig. 2(b) in red, which stems from the fact that the dislocation does not cross $p_{3}^{+}$along the path between the two neighboring minima. The choice in Fig. 1(b) is also convenient from the computational point of view because the same set of planes $p_{\alpha}^{ \pm}$is used for all states of the system along the path. A similar calculation can be made to estimate the position of the dislocation 


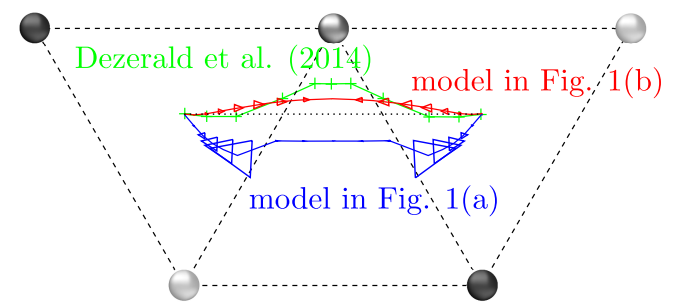

(a)

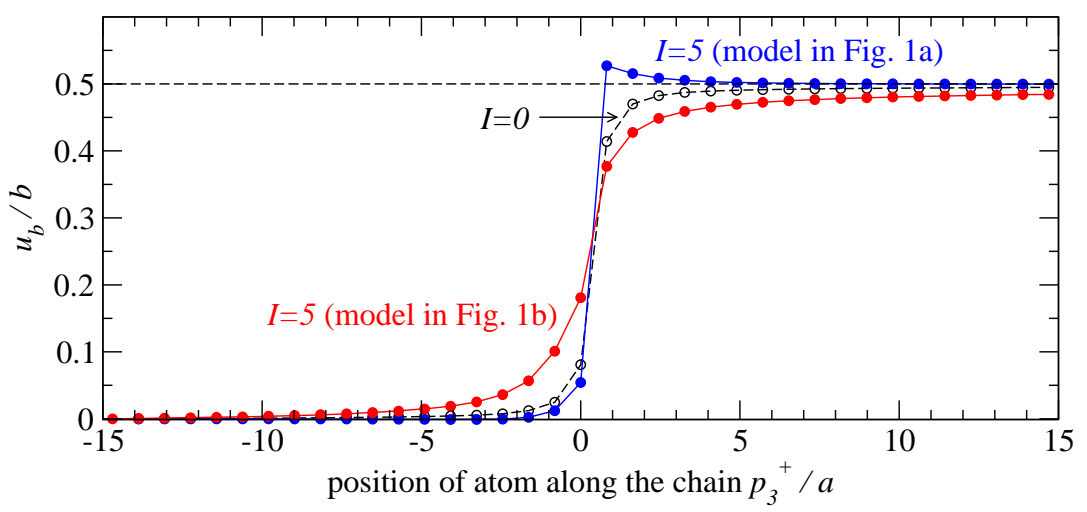

(b)

Figure 2: Performance of the model outlined in this paper, when the position of the dislocation is approximated from the six atomic chains shown in Fig. 1. Figure (a) shows the dislocation paths predicted using the choices of $p_{\alpha}^{ \pm}$shown in Fig. 1(a) and (b) and their comparison with the path obtained by Dezerald et al. in Ref. [27]. The nodes of the small triangles correspond to the intersections of line segments $Q_{\alpha}^{+} Q_{\alpha}^{-} \times Q_{\beta}^{+} Q_{\beta}^{-}$for the three possible slip planes $\alpha \neq \beta$ (see Fig. 1 for details). For each such small triangle, the position of the dislocation is associated with its centroid and the size of the triangle represents the error bar with which the position of the dislocation is calculated for a particular choice of the three sets of atomic chains $p_{\alpha}^{ \pm}$ in Fig. 1. Figure (b) shows the variation of the displacements $u_{b}$ along the atomic chains $p_{3}^{+}$in Fig. 1(a) and (b) drawn here by the blue and red lines, respectively.

when it moves on any of the other two $\{110\}$ planes of the [111] zone. This procedure is adopted for all the following calculations in this paper.

\section{Peierls barriers and dislocation glide}

Earlier in Ref. [19], we used the NEB+r method [18] and the Bond Order Potential (BOP) for BCC $\mathrm{W}[32]$ to investigate how the Peierls barrier of the $1 / 2[111]$ screw dislocation in tungsten depends on the applied stress. In these calculations, the dislocation was assumed to move between two neighboring sites along the straight line, which is a simplification that can now be circumvented. Our objective in the following is to use the calculated images that correspond to the minimum energy path of the system to: (i) identify the position of the dislocation in each image using the procedure formulated in the previous section, (ii) use these positions to determine the curved path of the dislocation on the three $\{110\}$ planes, and (iii) plot the energies of these snapshots along these curved paths to obtain the Peierls barriers. Within this model, the dislocation is assumed to move by elementary steps on the three $\{110\}$ planes of the [111] zone, which can give rise to an average (or macroscopic) slip on any plane of this zone.

We will first investigate how the shape of the dislocation pathway is affected by the shear stress perpendicular to the slip direction, $\tau$. We carry out these calculations for three representative values of this stress, namely $\tau / C_{44}=\{-0.04,0,0.04\}$ that is applied by imposing a uniform stress tensor $\boldsymbol{\Sigma} \equiv \boldsymbol{\Sigma}^{\text {nonglide }}=$ $\operatorname{diag}(-\tau, \tau, 0)$ in the coordinate system, where $x=[\overline{1} 2 \overline{1}], y=[\overline{1} 01]$ and $z=[111]$ (for details, see Ref. [19]). The calculated paths of the dislocation moving on the three $\{110\}$ planes of the [111] zone and the three 
values of the shear stresses perpendicular to the slip direction are shown in Fig. 3. Large triangles represent the boundaries of four dislocation sites - one from which the dislocation makes the jump (shaded) and the three others representing the target sites into which the dislocation moves by the glide on the three $\{110\}$ planes. The dislocation paths are obtained by piecewise linear interpolations of the dislocation positions obtained for each image by the method developed above and the small triangles represent the error bars with which the position of the dislocation was calculated.

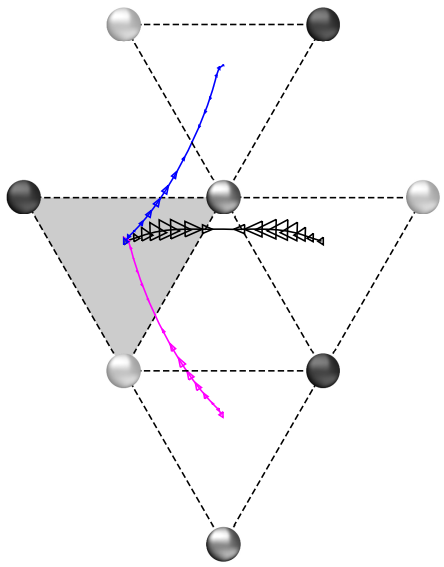

(a)

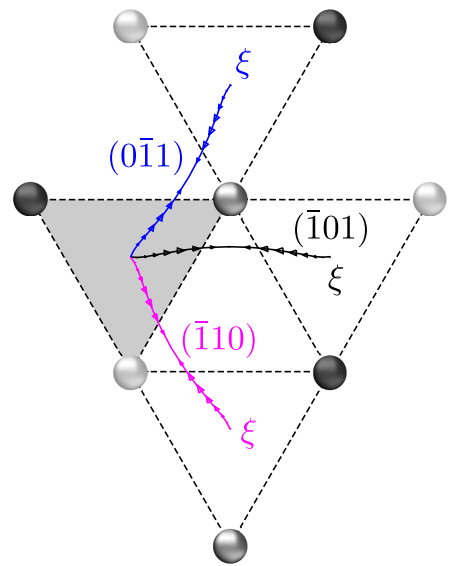

(b)

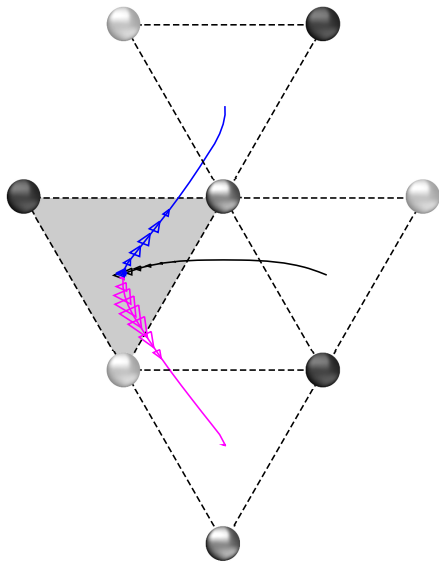

(c)

Figure 3: Dislocation pathways projected onto the (111) plane calculated from the positions of atoms in 15 movable images obtained using the NEB $+\mathrm{r}$ method. The three figures correspond to different shear stresses perpendicular to the slip direction: (a) $\tau / C_{44}=-0.04$, (b) $\tau / C_{44}=0$, (c) $\tau / C_{44}=0.04$. The calculated dislocation paths are shown by solid lines. The colors distinguish the paths of the dislocation on the three $\{110\}$ planes: $(\overline{1} 01)=$ black, $(0 \overline{1} 1)=$ blue, and $(\overline{1} 10)=$ purple. The middle panel shows the curvilinear coordinates $\xi$ for the three paths.

The Peierls barriers for the three $\{110\}$ planes and the three values of the shear stress perpendicular to the slip direction $(\tau)$ are shown in the upper panels of Fig. 4. Here, dashed curves correspond to the barriers obtained in Ref. [19], where we considered that the dislocation moves between the two minima along the straight path. In this case, the transition coordinate is defined as $\xi=a_{0} I /(M+1)$, where $I=\{0,1, \ldots, M+1\}$ are image numbers and $a_{0}=a \sqrt{2 / 3}$ the distance between neighboring minimum energy positions in $\{110\}$ planes $(a=3.1652 \AA)$. The solid curves are obtained by plotting $V\left(\xi ; \boldsymbol{\Sigma}^{\text {nonglide }}\right)$, obtained from (6), along the paths identified using the procedure developed in Section 3 and shown in Fig. 3. For $\tau=0$ the three barriers are identical, which is dictated by the symmetry. This is an important test of the procedure used to calculate the dislocation paths. The shape of the Peierls barrier is significantly different when drawn along the curved path than if following the straight line. In particular, the panels for zero and positive $\tau$ show that the barriers in black, that correspond to the glide of the dislocation on the $(\overline{1} 01)$ plane are wider, with steeper gradient close to $\xi=0$. The Peierls barrier thus deviates from the sinusoidal shape to a parabolic one or to a flat-top potential that has been often used to construct models of thermally activated dislocation glide [14, 28] (for a review, see the book of Caillard and Martin [33]). The right panel in Fig. 4 shows that the (011) and (110) barriers develop lower minima than the initial and final configurations along the path, which is a manifestation of the effect of the shear stress perpendicular to the slip direction.

For completeness, the lower panels of Fig. 4 show comparisons of the derivatives of these Peierls barriers. The curves in bold nearly satisfy the criterion $\sigma_{P} b=\max (\mathrm{d} V / \mathrm{d} \xi)$, where $\sigma_{P}=$ CRSS for the (101) plane and $\sigma_{P}=$ CRSS $/ 2$ for the (011) and (110) planes. Here, CRSS are the critical resolved shear stresses in the MRSSP (101) for which the dislocation starts to move in direct molecular statics calculations. The bold curves identify the $\{110\}$ plane on which the dislocation is predicted to move by these molecular statics calculations. In particular, the dislocation will move on the (101) plane for zero and positive $\tau$ (black curves), whereas the slip plane changes to (110) at negative $\tau$ (purple curves). The curvature of the path 


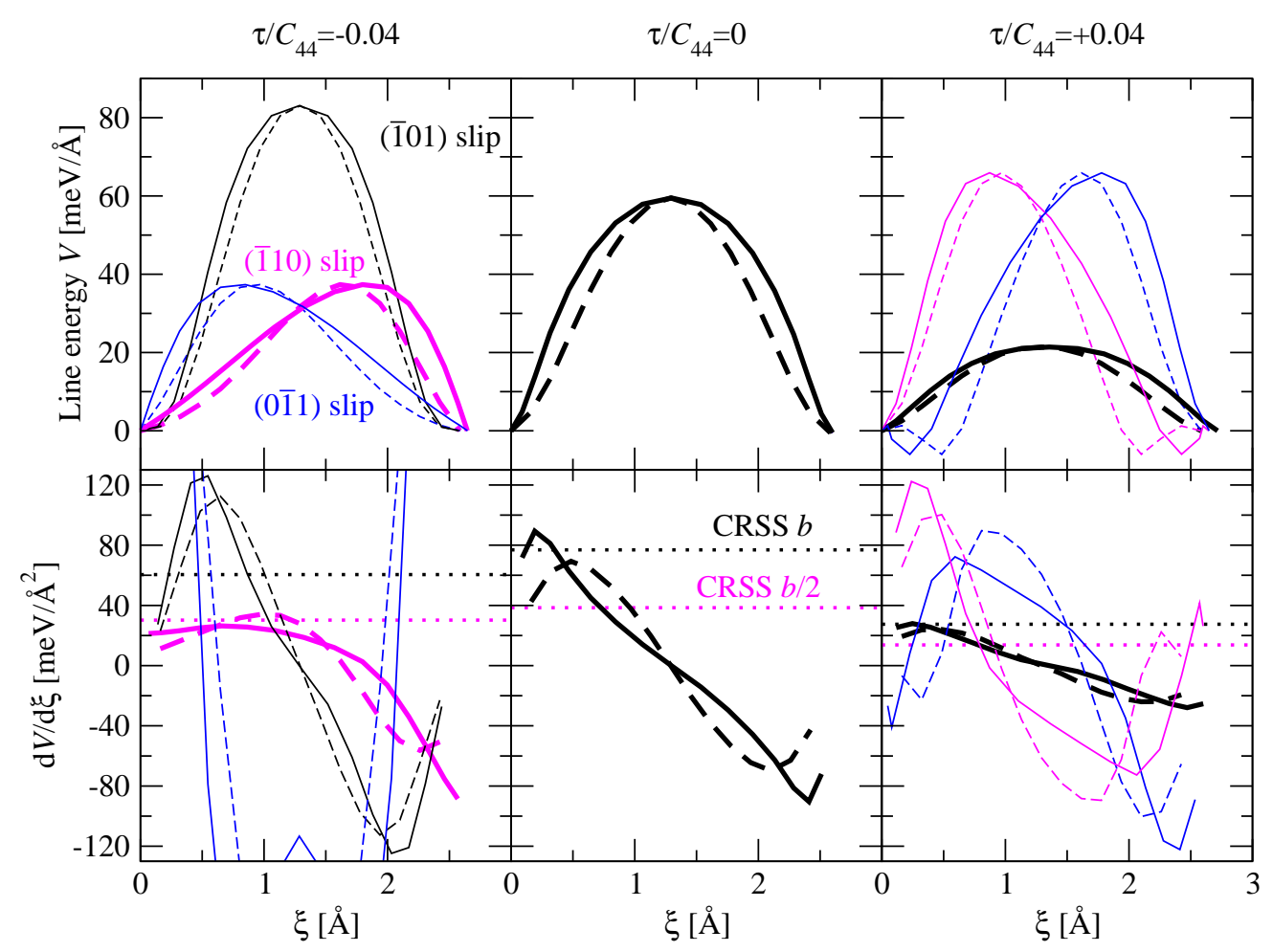

Figure 4: Comparison of the Peierls barriers of the 1/2[111] screw dislocation in BCC W determined using the images obtained from a series of $\mathrm{NEB}+\mathrm{r}$ calculations [19]. The barriers plotted by dashed lines are obtained by assuming that the dislocation moves along the straight line connecting the two neighboring potential minima in the glide plane. The solid curves are obtained by considering the curved path of the dislocation, as determined by the method developed in this paper and shown in Fig. 3 . For $\tau=0$ (middle panel), the Peierls barriers corresponding to dislocation glide on the three $\{110\}$ planes are identical. The bottom panel shows the derivatives of the Peierls barriers shown in the upper panels.

shifts the peak of $\max (\mathrm{d} V / \mathrm{d} \xi)$ towards the beginning of the path. This shift together with the steep increase of the Peierls barrier close to $\xi=0$ will affect the activation enthalpy of the dislocation glide [13]. This can be demonstrated easily in the limit of the zero applied stress for which the activated segment of the dislocation contains two isolated kinks between $\xi=0$ and $\xi_{\max }$ (the length of the curved transition path of the dislocation). The energy of this activated state relative to that of the straight dislocation at the bottom of the Peierls valley is obtained from the Dorn-Rajnak model [Eq. (6) in Ref. [14] with $\sigma^{*}=0$ and $\left.V\left(\xi_{0}\right)=V(0)=0\right]$ and reads

$$
2 H_{k}=2 \int_{0}^{\xi_{\max }} \sqrt{[V(\xi)]^{2}+2 E V(\xi)} \mathrm{d} \xi,
$$

where $H_{k}$ is the energy of an isolated kink, and $E$ the line tension of the dislocation at the bottom of the Peierls valley. Because both the shape of the Peierls barrier is known (see above) and the energy of two isolated kinks is determined experimentally [30] to be $2 H_{k}=2.06 \mathrm{eV}$, we can use Eq. (9) to determine $E$. For the Peierls barrier at $\tau=0$ plotted along the curved path (see the middle panel of Fig. 4 ), we obtained $E=3.17 \mathrm{eV} / \AA$. This value can be compared with the theoretical formula $E=\mu b^{2} / \alpha$, where $\mu$ is the $\{110\}\langle 111\rangle$ shear modulus defined as $\mu=\left(C_{11}-C_{12}+C_{44}\right) / 3$, to determine the value of $\alpha$. Using the data above, this analysis yields $\alpha=2.4$, which is not far from the value of 2 given in Ref. [24].

The curvature of the dislocation pathway and the associated flat maximum of the Peierls barrier are expected to result in larger curvature of the stress dependence of the activation enthalpy $[13,14]$ and thus to a steep increase of the flow stress with decreasing temperature, as observed universally in all BCC metals. To demonstrate the effect of the curvature of the dislocation pathway on these dependencies, we considered the loading in tension along [149] for which the temperature dependence of the flow stress was measured 
by Brunner [30]. For this loading, the maximum resolved shear stress plane (MRSSP) coincides with the (101) plane for which the Peierls barriers are shown in Fig. 4. During the tensile loading, both shear stresses perpendicular $(\tau)$ and parallel to the slip direction $(\sigma)$ increase proportionally to the applied load such that $\tau / \sigma=0.5$ and this ratio remains constant throughout the test. From our previous atomistic simulations, in particular from Fig. 7b in Ref. [9], we can see that for this particular character of loading, the value of the shear stress perpendicular to the slip direction at yield is $\tau / C_{44} \approx 0.02$. The Peierls barriers for this value of $\tau$ were calculated by the same method as above (not shown in Fig. 4). The dependence of the activation enthalpy on the applied shear stress parallel to the slip direction, $\sigma$, was then calculated as

$$
H_{b}(\sigma)=2 \int_{\xi_{0}}^{\xi_{c}} \sqrt{[E+V(\xi)]^{2}-\left[E+V\left(\xi_{0}\right)+\sigma b\left(\xi-\xi_{0}\right)\right]^{2}} \mathrm{~d} \xi,
$$

where the limits of integration are defined as $\sigma b=\mathrm{d} V /\left.\mathrm{d} \xi\right|_{\xi=\xi_{0}}$ and $V\left(\xi_{c}\right)-V\left(\xi_{0}\right)=\sigma b\left(\xi_{c}-\xi_{0}\right)$; for details, see Refs. [13,14]. Using the value of $E$ estimated above guarantees that $H_{b} \rightarrow 2 H_{k}$ as $\sigma \rightarrow 0$. However, for $H_{b}=0$, the stress at which the dislocation moves at $0 \mathrm{~K}(\sigma=2.48 \mathrm{GPa}$ from the Peierls barrier for the straight and $2.92 \mathrm{GPa}$ for the curved dislocation pathway) is roughly a factor of 3 higher than the value obtained by extrapolating the experimental flow stress to $0 \mathrm{~K}$ (about 1 GPa from (author?) [30]). This discrepancy is well known [34-40] and can be removed by rescaling all stresses by a constant value so as to bring the Peierls stress at $0 \mathrm{~K}$ into agreement with the experiment. This rescaled dependence of $H_{b}(\sigma)$ can be used to obtain the temperature dependence of the flow stress from the standard Arrhenius law for the plastic strain rate, $\dot{\gamma}=\dot{\gamma}_{0} \exp \left[-H_{b}(\sigma) / k_{B} T\right]$, where $\dot{\gamma}_{0}$ is the maximum plastic strain rate corresponding to an unbound dislocation. In particular, this can be written as $H_{b}(\sigma)=q k_{B} T$, where $q=\log \left(\dot{\gamma}_{0} / \dot{\gamma}\right)$ depends on the experimental strain rate. Here, $q$ can be obtained also from the condition $2 H_{k}=q k_{B} T_{k}$, where $T_{k}$ is the temperature at which the thermal component of the flow stress vanishes in the experiment (i.e. the activation enthalpy rises to its maximum, $2 H_{k}$ ). In this equation, the influence of the plastic strain rate enters through the value of $T_{k}$ that can be read-off from experiments. From the measurements of Brunner [30] done at $\dot{\gamma}=8.5 \times 10^{-4} \mathrm{~s}^{-1}$, we obtained $T_{k}=760 \mathrm{~K}$ and thus the equation above yields $q=31.4$.

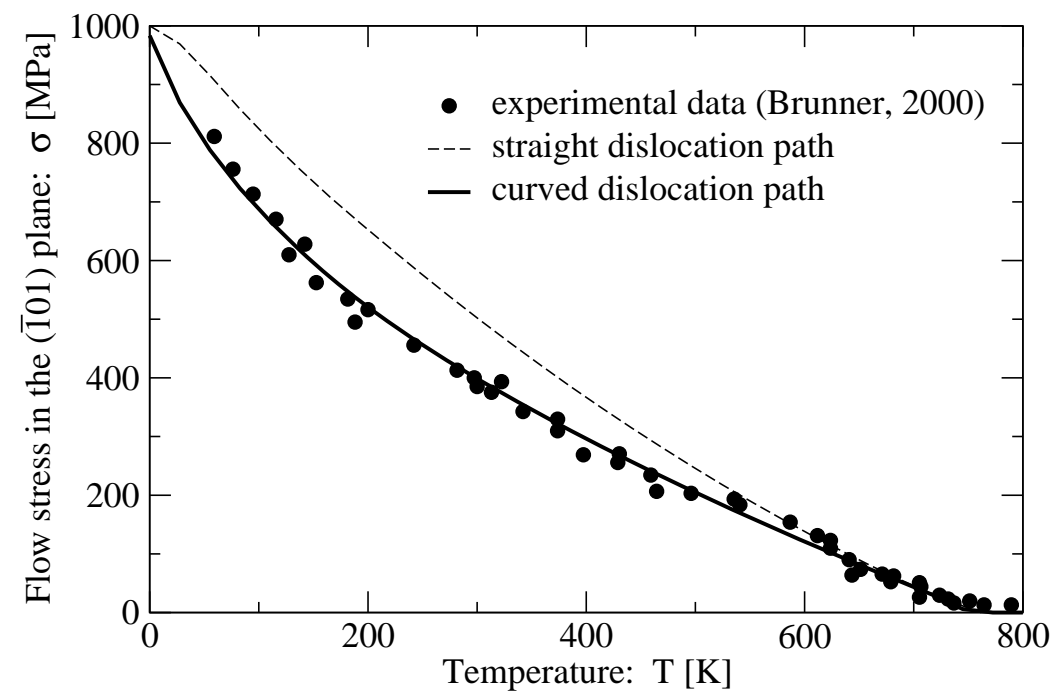

Figure 5: Temperature dependence of the flow stress predicted by considering a straight dislocation path (dashed curve) and the curved path obtained by the procedure developed in this paper (solid curve). The experimental data for loading in tension along [149] obtained by Brunner [30] are shown by circles.

The temperature dependence of the flow stress obtained by considering straight and curved dislocation paths for the loading in tension along [149] are shown in Fig. 5. These are compared with the experimental measurements of Brunner [30] depicted by circles. One can clearly see that neglecting the curvature of the dislocation path leads to significant deviation of the predicted yield stress from experimental values, 
in particular at intermediate and low temperatures. On the other hand, for the Peierls barrier determined along the curved dislocation path, the experimental measurements are very well reproduced throughout the whole range of temperatures. Since the calculations of the Peierls barriers and the corresponding paths do not depend on the details of interatomic forces used, it is reasonable to expect that the significance of the curved paths will be found generally.

\section{Conclusions}

We have developed a numerical scheme that specifies the position of the center of the dislocation only from the knowledge of the displacements of atoms between the relaxed configuration and the perfect lattice. This represents a generalization of the model devised originally by Peierls and Nabarro [20, 21]. Our model goes beyond these developments in that it provides two components of the dislocation position, one of which lies in the glide plane, whereas the other is perpendicular to this plane.

The position of the dislocation in each of the three possible $\{110\}$ slip planes is identified as the point where the displacement of atoms parallel to the slip direction is half-way between its minima and maxima. This calculation is made separately for the chain of atoms above and below the triangular site containing the dislocation. For each slip plane, one thus obtains two points that define a line segment which represents all possible positions of the dislocation. The pairwise intersections of the three lines thus obtained define the corners of a triangle that approximates the position of the dislocation. We have demonstrated which atomic chains around the dislocation site should be considered to obtain an accurate representation of the dislocation pathway. This method differs from the existing methods [23] in that we determine the position of the dislocation only from the positions of atoms in the dislocation core, while the elastically displaced atoms further away do not play any direct role. This is very convenient because the atoms in far-field are often influenced by the mismatch between the actual position of the dislocation (moved around by the NEB forces) and the initially interpolated (and later fixed) anisotropic elastic strain field at the boundary of the simulated block in the direction perpendicular to the dislocation.

We have demonstrated the performance of this procedure by calculating the path of the dislocation between two minimum energy positions, using the snapshots of atomic positions obtained from our recent $\mathrm{NEB}+\mathrm{r}$ calculations made in Ref. [19]. These calculations have been made for all three $\{110\}$ planes on which the dislocation can move and for zero, positive and negative shear stresses perpendicular to the slip direction. The curvature of the dislocation path is shown to affect the shape of the Peierls barrier, which becomes steeper close to the beginning and end of the transition path and, at the same time, more flat close to its maximum. We have demonstrated the importance of the curvature of the dislocation pathway in a line tension dislocation model for thermally activated formation of pairs of kinks by calculating the stress dependence of the activation enthalpy for a hypothetical straight and the actual curved dislocation path. These results provide the predictions of the temperature dependence of the flow stress that is compared here with the experimental measurements of Brunner [30]. This comparison shows that neglecting the curvature of the dislocation path leads to predictions that deviate significantly from experimental measurements. On the other hand, combining the NEB calculations of the energies of individual snapshots of the system with the method of determination of the curved dislocation pathway, developed in this paper, yields the temperature dependence of the flow stress that is in very good agreement with the experiment [30].

The method of calculation of the curved dislocation pathway, developed in this paper, should be applicable to all materials with BCC crystal structure the plastic deformation of which is controlled by $1 / 2\langle 111\rangle$ screw dislocations. Moreover, the idea of approximating the dislocation position from several different planes containing the Burgers vector of the dislocation should be applicable also to other crystal structures with non-cubic symmetry, most notably HCP metals some of which deform by thermally activated dislocation glide.

\section{Acknowledgments}

This research was supported by the Marie-Curie International Reintegration Grant No. 247705 "MesoPhysDef", by the Academy of Sciences of the Czech Republic, Project no. RVO:68081723 (RG) and by the 
US Department of Energy, BES, Grant no. DE FG02-98ER45702 (VV). This work has been carried out at the Central European Institute of Technology (CEITEC) with research infrastructure supported by the project CZ.1.05/1.1.00/02.0068 financed from the EU Structural Funds.

[1] V. Vitek, R. C. Perrin, D. K. Bowen, Core structure of $1 / 2\langle 111\rangle$ screw dislocations in b.c.c. crystals, Philos. Mag. 21 (173) (1970) 1049-1073.

[2] W. Xu, J. A. Moriarty, Accurate atomistic simulations of the Peierls barrier and kink-pair formation energy for $\langle 111\rangle$ screw dislocations in bcc Mo, Comp. Mater. Sci. 9 (1998) 348-356.

[3] C. Woodward, S. I. Rao, Ab-initio simulation of isolated screw dislocations in bcc Mo and Ta, Philos. Mag. A 81 (5) (2001) 1305-1316.

[4] S. L. Frederiksen, K. W. Jacobsen, Density functional theory studies of screw dislocation core structures in bcc metals, Philos. Mag. 83 (3) (2003) 365-375.

[5] J. Li, C.-Z. Wang, J.-P. Chang, W. Cai, V. V. Bulatov, K.-M. Ho, S. Yip, Core energy and Peierls stress of a screw dislocation in bcc molybdenum: A periodic-cell tight-binding study, Phys. Rev. B 70 (2004) 104113.

[6] M. Cawkwell, D. Nguyen-Manh, C. Woodward, D. G. Pettifor, V. Vitek, Origin of brittle cleavage in iridium, Science 309 (2005) 1059-1062.

[7] L. Ventelon, F. Willaime, Core structure and Peierls potential of screw dislocations in $\alpha$-Fe from first principles: cluster versus dipole approaches, J. Comp.-Aid. Mat. Design 14 (2007) 85-94.

[8] L. Pizzagalli, P. Beauchamp, H. Jónsson, Calculations of dislocation mobility using Nudged Elastic Band method and first principles DFT calculations, Philos. Mag. 88 (1) (2008) 91-100.

[9] R. Gröger, A. G. Bailey, V. Vitek, Multiscale modeling of plastic deformation of molybdenum and tungsten: I. Atomistic studies of the core structure and glide of $1 / 2\langle 111\rangle$ screw dislocations at 0 K, Acta Mater. 56 (2008) 5401-5411.

[10] D. Rodney, L. Proville, Stress-dependent Peierls potential: Influence on kink-pair activation, Phys. Rev. B 79 (2009) 094108.

[11] M. Mrovec, D. Nguyen-Manh, C. Elsässer, P. Gumbsch, Magnetic bond-order potential for iron, Phys. Rev. Lett. 106 (2011) 246402.

[12] V. Celli, M. Kabler, T. Ninomiya, R. Thomson, Theory of dislocation mobility in semiconductors, Phys. Rev. 131 (1) (1963) 58-72.

[13] J. E. Dorn, S. Rajnak, Nucleation of kink pairs and the Peierls' mechanism of plastic deformation, Trans. AIME 230 (1964) 1052-1064.

[14] R. Gröger, V. Vitek, Multiscale modeling of plastic deformation of molybdenum and tungsten: III. Effects of temperature and plastic strain rate, Acta Mater. 56 (2008) 5426-5439.

[15] V. V. Bulatov, E. Kaxiras, Semidiscrete variational Peierls framework for dislocation core properties, Phys. Rev. Lett. 78 (22) (1997) 4221-4224.

[16] H. Jónsson, G. Mills, K. W. Jacobsen, Classical and Quantum Dynamics in Condensed Phase Simulations, World Scientific, 1998, Ch. 16. Nudged elastic band method for finding minimum energy paths of transitions, pp. 385-404.

[17] G. Henkelman, H. Jónsson, Improved tangent estimate in the nudged elastic band method for finding minimum energy paths and saddle points, J. Chem. Phys. 113 (22) (2000) 9978-9985.

[18] R. Gröger, V. Vitek, Constrained Nudged Elastic Band calculation of the Peierls barrier with atomic relaxations, Model. Simul. Mater. Sci. Eng. 20 (2012) 035019.

[19] R. Gröger, V. Vitek, Stress dependence of the Peierls barrier of $1 / 2\langle 111\rangle$ screw dislocations in BCC metals, Acta Mater. 61 (2013) 6362-6371.

[20] R. Peierls, The size of a dislocation, Proc. Phys. Soc. 52 (1) (1940) 34-37.

[21] F. R. N. Nabarro, Dislocations in a simple cubic lattice, Proc. Phys. Soc. 59 (2) (1947) 256-272.

[22] L. Proville, L. Ventelon, D. Rodney, Prediction of the kink-pair formation enthalpy on screw dislocations in $\alpha$-iron by a line tension model parametrized on empirical potentials and first-principles calculations, Phys. Rev. B 87 (2013) 144106.

[23] L. Ventelon, F. Willaime, E. Clouet, D. Rodney, Ab initio investigation of the Peierls potential ofscrew dislocations in bcc Fe and W, Acta Mater. 61 (11) (2013) 3973-3985.

[24] J. P. Hirth, J. Lothe, Theory of dislocations, 2nd Edition, J.Wiley \& Sons, New York, 1982.

[25] M. Itakura, M. Kaburaki, M. Yamaguchi, First-principles study on the mobility of screw dislocations in bcc iron, Acta Mater. 60 (2012) 3698-3710.

[26] R. Heinrich, W. Schellenberger, Nonequilibrium configurations and Peierls potential of screw dislocations in the B.C.C. lattice, Phys. Stat. Sol. B 47 (1971) 81-92.

[27] L. Dezerald, L. Ventelon, E. Clouet, C. Denoual, D. Rodney, F. Willaime, Ab initio modeling of the two-dimensional energy landscape of screw dislocations in bcc transition metals, Phys. Rev. B 89 (2014) 024104.

[28] T. Suzuki, H. Koizumi, H. O. K. Kirchner, Plastic flow stress of b.c.c. transition metals and the Peierls potential, Acta Metall. Mater. 43 (6) (1995) 2177-2187.

[29] R. Gröger, Development of physically based plastic flow rules for body-centered cubic metals with temperature and strain rate dependencies, Ph.D. thesis, University of Pennsylvania (2007). URL http://arxiv.org/abs/0707.3577

[30] D. Brunner, Comparison of flow-stress measurements on high-purity tungsten single crystals with the kink-pair theory, Mater. Trans. 41 (1) (2000) 152-160.

[31] G. J. Ackland, R. Thetford, An improved n-body semi-empirical model for body-centred cubic transition metals, Philos. Mag. A 56 (1) (1987) 15-30.

[32] M. Mrovec, R. Gröger, A. G. Bailey, D. Nguyen-Manh, C. Elsässer, V. Vitek, Bond-order potential for simulations of 
extended defects in tungsten, Phys. Rev. B 75 (2007) 104119.

[33] D. Caillard, J. L. Martin, Thermally activated mechanisms in crystal plasticity, Pergamon Press, 2003.

[34] Z. S. Basinski, M. S. Duesbery, G. S. Murty, The orientation and temperature dependence of plastic flow in potassium, Acta Metall. 29 (5) (1981) 801-807.

[35] W. Pichl, M. Krystian, The plasticity of potassium, Mat. Sci. Eng. A234-236 (1997) 426-429.

[36] M. Wen, A. H. W. Ngan, Atomistic simulation of kink-pairs of screw dislocations in body-centred cubic iron, Acta Mater. 48 (2000) 4255-4265.

[37] C. Woodward, S. I. Rao, Flexible ab initio boundary conditions: Simulating isolated dislocations in bcc Mo and Ta, Phys. Rev. Lett. 88 (21) (2002) 216402.

[38] R. Gröger, V. Vitek, Explanation of the discrepancy between the theoretical and measured yield stresses in body-centered cubic metals, Philos. Mag. Lett. 87 (2) (2007) 113-120.

URL http://arxiv.org/abs/cond-mat/0605449

[39] L. Proville, D. Rodney, M.-C. Marinica, Quantum effect on thermally activated glide of dislocations, Nature Mater. 11 (2012) 845.

[40] D. Caillard, On the stress discrepancy at low-temperatures in pure iron, Acta Mater. 62 (2014) 267-275. 